\title{
Downregulation of Dual-specificity phosphatase 9 promotes tumor progression and contributes to poor prognosis in colorectal cancer
}

\section{Zhaoyan Qiu ( $\sim 864093026 @ q q . c o m$ )}

PLAGH

Ning Liang

The 75th Group Army Hospital

Tao Sun

Zhengzhou University

Hongyuan Xue

Fudan University

Tianyu Xie

PLAGH

Xinxin Wang

PLAGH

Qian Wang

Zhengzhou University

\section{Research}

Keywords: DUSP9, colorectal cancer, CpG island, bisulfite sequencing, prognosis

Posted Date: February 11th, 2020

DOI: https://doi.org/10.21203/rs.2.23210/v1

License: (a) This work is licensed under a Creative Commons Attribution 4.0 International License. Read Full License 


\section{Abstract}

Background Dual-specificity phosphatase 9 (DUSP9) belongs to the dual-specificity protein phosphatase subfamily. Recently, increasing attention has been paid on the role of DUSP9 in a variety of cancers. However, its functional role in tumor development is still unclear, especially in colorectal cancer (CRC).

Methods The functional role of DUSP9 in inhibiting the progression of CRC was verified both in vivo and in vitro using colony formation assay, EdU incorporation assay, wound healing assay, nude mice xenograft model, and et al. RNA-seq was performed to assess the gene expression profiling in SW480 cells with DUSP9 stable knockdown and shControl cells. Bisulfite sequencing (BSE) was performed to reveal methylation status of $\mathrm{CpG}$ island in promoter of DUSP9.

Results DUSP9 was significantly down regulated in tumor tissues compared with peritumor tissues. Moreover, low DUSP9 expression in CRC was closely associated with tumor size, depth of invasion and advanced TNM stage, indicating that DUSP9 may be involved in the progression of CRC. Kaplan-Meier survival analysis showed that the overall survival (OS) and recurrence-free survival (RFS) of patients with low expression of DUSP9 were significantly shorter than that of patients with high expression of DUSP9. Functional study revealed that DUSP9 inhibited tumor migration, invasion and metastasis both in vitro and in vivo. Mechanistically, low expression of DUSP9 in CRC was caused by the upregulation of miR1246 and hypermethylation status of CpG island in promoter of DUSP9.

Conclusion Our findings demonstrate that DUSP9 plays a critical role in the progression of CRC and therapeutic intervention to increase the expression or activity of DUSP9 may be a potential target for CRC treatment in the future.

\section{Background}

Colorectal cancer (CRC) is a malignancy with high incidence in digestive system. According to statistics, there are about 1.2 million new cases and 600000 deaths in the world every year ${ }^{1}$. With the changing of population and dietary habit throughout the world, the number of new cases is still on the rise every year, and the age of onset is getting younger and younger ${ }^{2}$. The recognized risk factors for CRC include dietary factors (such as high animal fat, high animal protein, high energy and low cellulose diet with more westernized lifestyle), genetic factors (especially familial adenomatous polyposis and hereditary nonpolyposis), and disease factors (such as colorectal adenoma, ulcerative colitis, polyposis) ${ }^{3}$. At present, surgical resection, radiotherapy, chemotherapy and biotherapy are the main treatments, but the effect is very limited. Moreover, the specificity and sensitivity of serum markers (such as CEA and CA199) in the diagnosis of colon cancer are difficult to achieve satisfactory results ${ }^{4}$. Therefore, it is very important to screen and identify new key molecules that involved in the initiation and progression of CRC.

Dual-specificity phosphatase 9 (DUSP-9), also known as MKP-4 (mitogen-activated protein kinase phosphatase 4), belongs to the dual-specificity protein phosphatase subfamily, which has been reported 
to dephosphorylate tyrosine and threonine/serine residues of their substrates ${ }^{5}$. Recently, it was observed that DUSP9 plays a critical role in the normal function of placenta, whereas is not required for mammalian embryonic development ${ }^{6}$. In addition, Ye et al. demonstrated that DUSP9 inhibits the progression of nonalcoholic fatty liver disease (NAFLD) through ASK1 suppression, suggesting that Dusp9 may be an ideal therapeutic target for the treatment of NAFLD ${ }^{7}$. Recently, increasing attention has been paid on the role of DUSP9 in a variety of cancers. Accumulating evidence suggests that DUSP9 is down- regulated and acts as a tumor suppressor in many kinds of cancers, such as gastric cancer, hepatocellular carcinomas, renal cancer, squamous cell carcinoma and et al ${ }^{8-11}$. Moreover, in a mouse model, DUSP9 has also been found to play an anti-tumor role in squamous cell carcinoma and non-small cell lung cancer (NSCLC) ${ }^{12}$. However, as far as we know, the expression pattern of DUSP9 in CRC has not been reported. Additionally, the function of DUSP9 in CRC and its regulatory mechanism are still unclear.

In the present study, we systematically investigated the expression and functional roles of DUSP9 in CRC as well as its clinical implication. This study provides a new dimension to understand the pathological roles of DUSP9 in CRC development and provides experimental evidence for DUSP9 as a potential therapeutic target in CRC.

\section{Materials And Methods}

\section{Cell culture and tissue collection}

Human CRC cell lines SW480 and 293T were purchased from ATCC (Manassas, VA, USA), which cultured in RPMI-1640 medium (Gibco) containing with $10 \%$ serum. A total of 245 paired CRC tissues were collected at Chinese PLA general hospital in the present study, which was approved by the Ethics Committee of Chinese PLA general hospital. Moreover, all participants involved in this study have signed informed consent. For the clinical data, please see Table 1.

\section{Cell viability assay}

MTS assay (G3581; Promega Corporation, Madison, WI) was used for cell viability detectin. The absorbance was measured at 490nm using a spectrophotometer.

\section{Colony formation assay}

The stable transfected CRC cells (SW480 and 293T) were seeded in six-well plates with the density of $1 \times$ $10^{3} /$ well. After 2 weeks, CRC cells were fixed in $70 \%$ ethanol and then stained with $5 \%$ crystal violet. Count the colony numbers under the microscope.

\section{EdU incorporation assay}

The EDU reagent was diluted to $5 \mu \mathrm{m}$ in serum-free medium and added to the cells for $2 \mathrm{~h}$. After PBS cleaning, $4 \%$ paraformaldehyde was added for $30 \mathrm{~min}$ and then $0.5 \%$ Triton X-100 was added for 20 min. 
Dye these cells with Apollo ${ }^{\circledR}$ reaction cocktail according to the instructions. The prepared Hoechst 33342 solution was added to stain the nucleus for 30 minutes. Finally, positive cells were counted.

\section{Wound healing assay}

Wound healing assay was performed to assess cell migration ability. In short, three scratch wounds in each well were made using plastic pipette tips and the wound closure were observed at 0 and $48 \mathrm{~h}$.

\section{Nude mice xenograft model}

Six to eight weeks-old BALB/c nude mice were randomly divided into experimental group and control group. The establishment of mice xenograft model refers to previous methods. The calculation formula of tumor volume is as follows: $V=1 / 2 \times L \times W^{2}$ ( $L$, the longest dimension; $W$, shortest dimension). This study was approved by the Ethics Committee of Chinese PLA general hospital for animal research.

\section{Western blot and immunohistochemical staining}

Western blot and immunohistochemistry staining were performed as previously described ${ }^{13}$. For western blot assay, the primary antibodies against DUSP9 (1:2000, Abcam, cat. no. ab167080), E-cadherin (1;2000, Abcam, cat. no. ab194982), ZO-1 (1;1500, Abcam, cat. no. ab221547), Vimentin (1:2000, Abcam, cat. no. 92547), N-cadherin (1;1000, Abcam, cat. no. ab18203), and $\beta$-actin (1:3000, Abcam, cat. no. 179467) were used in accordance with the manufacturer's instructions. Signals were detected using an ECL kit (Pierce, Rockford, IL) as previously reported ${ }^{13}$. For immunohistochemistry staining, primary antibodies against DUSP9 (1:200, Abcam, cat. no. ab167080), and PCNA (1:1000, Abcam, cat. no. ab92552) were used according to the manufacturer's instructions. The IHC scoring methods were as follows: (1) the staining intensity of positive cells (recorded as A): 0 for non staining, 1 for light staining, 2 for moderate staining and 3 for strong staining; (2) The percentage of positive cells (recorded as B); 0 for $0-10 \%, 1$ for $11 \%-25 \%, 2$ for $26 \%-50 \%, 3$ for $51 \%-75 \%$, and more than $75 \%$ is 4 points. (3) The product of $A$ and $B$ is the final IHC score. Semi quantitative analysis method: $0-1$ for,$- 2-4$ for,$+ 5-8$ for,$++ 9-12$ for +++ .

\section{RNA-seq analysis}

The RNA-seq technique was performed to assess the gene expression profiling in SW480 cells with DUSP9 stable knockdown and SW480-shControl cells. Trizol Kit (Promega, USA) was used to extract the total RNA of the above samples. The cDNA fragments were purified and enriched by PCR to construct the cDNA library. Finally, the cDNA library was sequenced on the Illumina sequencing platform (IlluminaHiSeq ${ }^{\text {TM }}$ 2500).

\section{Bisulfite sequencing}

Bisulfite sequencing was performed as previously described ${ }^{14}$. Briefly, CRC and normal intestinal mucosa samples were digested with proteinase $\mathrm{K}$ to extract sample DNA, which was subject to the bisulfite 
reaction using an EpiTect Bisulfte kit (Qiagen, Germany). Modified DNA was amplified by PCR and then examined by gel electrophoresis. The BLAST program of the National Center for Biotechnology Information was used to identify the sequence homologies.

\section{Statistical analysis}

All statistical analyses were performed using SPSS 17.0 software (SPSS, Chicago, IL). Two-sided Student's $t$ test was used to examine differences between two groups. Pearson correlation analyses were used to assess the correlations between measured variables. Kaplan-Meier survival curve was plotted for OS and RFS and compared by log-rank test. $P$ values $<0.05$ were considered statistically significant.

\section{Results}

\section{DUSP9 is down regulated in CRC tissues and associated with tumor progression and poor prognosis}

To assess the role of DUSP9 in human CRC, we first evaluated the protein expression levels of DUSP9 in 18 paired CRC tissues by western blot. Our results demonstrated that DUSP9 was significantly down regulated in CRC tissues compared with peritumor tissues (Fig. 1A). The results of protein quantification were shown in Fig.1B. In addition, quantitative real-time reverse transcription (qRT-PCR) analysis showed that DUSP9 was down regulated in CRC tissues at mRNA level (Fig. 1C). As shown in Fig. 1D, immunohistochemical staining analysis in CRC tissues from another cohort of 245 patients further confirmed the downregulation of DUSP9 at the protein level in CRC tissues compared with paired peritumor tissues. Finally, we explored the clinical relevance of DUSP9 expression. Kaplan-Meier survival analysis showed that the overall survival (OS) and recurrence-free survival (RFS) of patients with low expression of DUSP9 were significantly shorter than that of patients with high expression of DUSP9 (Fig. 1E, F). Moreover, low DUSP9 expression in CRC was closely associated with tumor size, depth of invasion and advanced TNM stage, indicating that DUSP9 may be involved in the progression of CRC (Table 1). In addition, DUSP9 expression, depth of invasion and TNM stage were found to be associated with overall survival (OS) and disease-free survival (DFS) of these patients in univariate survival analysis. Multivariate analysis indicated that DUSP9 expression could be a prognostic factor for OS and DFS of patients with CRC after adjusting for gender, age at diagnosis, depth of invasion and TNM stage, indicating that DUSP9 may be an independent prognostic factor for CRC (Supplementary Table 1 and 2).

\section{RNA-seq revealed the pathways that DUSP9 inhibited proliferation and metastasis in CRC}

To determine the biological function of DUSP9 in CRC, we performed RNA-seq analysis of significantly differently expressed genes (DEGs) between DUSP9 stable knockdown-SW480 cells and shControl cells. Of the 4096 dysregulated genes between two groups, 2113 genes were up-regulated (fold change $\geq 2$ ) and 1983 genes were down-regulated (fold change $\geq 2$ ) (Fig. 2A). Part of representative dysregulated genes between SW480 cells with DUSP9 stable knockdown and SW480-shControl cells are listed in Supplemental Table 3. In order to explore the role of DURP9 in tumor progression, we performed KEGG pathway analysis of these DEGs between two groups, and the results showed that many tumor growth 


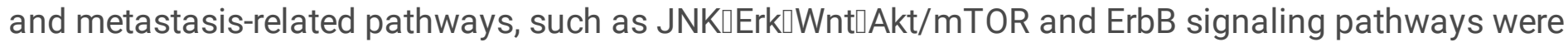
significantly enriched in DUSP9 knockdown group. This suggests that DUSP9 knockdown can activate tumor growth and metastasis-related pathways (Fig. 2B). A hierarchical cluster of DEGs is partially shown in Fig. 2C. As shown in the heatmap, mesenchymal markers ( $\mathrm{N}$-cadherin and vimentin) were significantly increase and epithelial markers (E-cadherin and zonula occludens-1) were remarkablely decrease in shDUSP9 SW480 cells than control cells, indicating that DUSP9 knockdown significantly promotes the progression of CRC. Moreover, GSEA (Gene Set Enrichment Analysis) also showed that downregulation of DUSP9 led to the activation of Erk and Akt/mTOR signaling pathway (Fig. 2D).

\section{DUSP9 suppresses tumor growth in vivo}

In order to further verify the tumorigenicity of DUSP9 in vivo, we constructed a tumor model of nude mice using SW480 and 293T CRC cell lines with stable overexpression or knockdown of DUSP9. These cells were then transplanted into nude mice to test their tumorigenicity in vivo. Subcutaneous tumor growth was monitored every 3 days, and these mice were euthanized after 30 days. Tumors derived from SW480 cells with stable DUSP9

knockdown showed an increased growth rate and less net weight at the fourth week when compared with control mice (Fig. 3A). In contrast, the tumor growth rate was slower, and the average tumor weight was significantly reduced in mice inoculated with SW480 cells with stable DUSP9 overexpression at the fourth week when compared with control mice (Fig. 3B).

Moreover, when compared with controls, the xenografts developed from SW480 cells with DUSP9 stable knockdown exhibited a significant increase of positive PCNA staining. However, overexpression of DUSP9 exhibited a considerable decrease of positive PCNA staining in xenografts developed from 293T cells (Fig. 3C, D). In conclusion, the above results further confirmed the antitumor effect of DUSP9 on the progression of CRC.

\section{Enforced expression of DUSP9 inhibits proliferation of CRC cells}

Next, a variety of in vitro assays were carried out with loss-of-function or gain-of-function of DUSP9 to evaluate the potential role of DUSP9 on CRC cell functions. The results of MTS cell viability assay showed that SW480 cells with DUSP9 stable knockdown had a faster growth rate than control cells, whereas 293T cells with DUSP9 stable overexpression had a slower growth rate than control cells (Fig. 4A). Colony forming assays revealed that down-regulation of DUSP9 significantly promoted cell proliferation of SW480 cells, whereas up-regulation of DUSP9 significantly inhibited cell proliferation of 293 T cells (Fig. 4B). Similarly, the number of 5-ethynyl-2'-deoxyuridine (EdU) incorporation was significantly increased in SW480 cells with DUSP9 knockdown, but were markedly decreased in 293T cells with DUSP9 overexpression compared with controls (Fig. 4C). These results demonstrated that DUSP9 functioned as a key regulatory factor in cell proliferation of CRC.

\section{DUSP9 inhibits tumor migration, invasion and metastasis in vitro}


We next investigated the effects of DUSP9 on the migration, invasion and metastasis of CRC cells. The scratch wound healing assays showed that knockdown of DUSP9 significantly promoted the migratory ability of SW480 cells. In contrast, DUSP9 overexpression remarkablely inhibited the migratory ability of 293 T cells (Fig. 5A). Accordingly, the transwell invasion assay showed that DUSP9 overexpression significantly impaired the invasion of 293T cells. However, knockdown of DUSP9 in SW480 cells acted the opposite way (Fig. 5B). It is well established that the epithelial-mesenchymal transition (EMT) plays a key role in tumor metastasis by increasing cell mobility and reducing cell-cell contact. We further investigated whether EMT is involved in DUSP9 mediated invasion and metastasis of CRC cells. Western blot analysis and quantitative RT-PCR showed that mesenchymal markers (N-cadherin and vimentin) were significantly increase and epithelial markers (E-cadherin and zonula occludens-1) were remarkablely decreased when DUSP9 were knockdown in SW480 cells. However, the opposite effect was observed following DUSP9 overexpression in 293T cells (Fig. 5C, D). These findings indicated that DUSP9 inhibited tumor migration, invasion and metastasis in CRC.

\section{miR-1246 promotes the proliferation and invasion of CRC cells by inhibiting DUSP9}

Accumulating evidence has suggested that MicroRNA is critical to the regulation of gene-expression network and is frequently dysregulated in many types of cancers. In this study, MicroRNA Data Integration Portal (mirDIP)-based target prediction programs were used to identify the potential microRNAs involved in the downregulation of DUSP9 in CRC. The top ten predicted miRNAs targeting DUSP9 were listed in Fig. 6A. Real-time PCR and Western blot showed that miR-1246 remarkablely reduced DUSP9 expression in SW480 and 293T cells (Fig. 6B, C). On the contrary, micro-1246 inhibition can elevate the expression of DUSP9 both in mRNA and protein levels (Fig. 6D, E). Moreover, a significant negative correlation $(r=-0.642, p<0.01)$ between miR-1246 and DUSP9 was found in tumor tissues from $30 \mathrm{CRC}$ patients (Fig. 6F). In addition, miR-1246 mimics attenuated the ability of DUSP9 to inhibit the proliferation of CRC cells, whereas miR-1246 inhibition decreased the proliferation by DUSP9 knockdown in CRC cells (Fig. 6G). Moreover, transwell invasion experiment revealed that miR-1246 mimics attenuated the ability of DUSP9 to inhibit the invasion of CRC cells (Fig. 6H). Altogether, these results suggest that miR-1246 promotes the proliferation and invasion of CRC cells by inhibiting DUSP9.

\section{DUSP9 expression is silenced via promoter hypermethylation in CRC}

In order to further explore the reason for the decrease of DUSP9 in CRC, we used MethPrimer software to predict the methylation status in DUSP9 gene promoter. The results showed that DUSP9 was hypermethylated in a variety of cancers, such as colon adenocarcinoma (COAD), bladder urothelial carcinoma (BLCA), breast invasive carcinoma (BRCA), cervical squamous cell carcinoma and endocervical adenocarcinoma (CESC), lung adenocarcinoma (LUAD) and pancreatic adenocarcinoma etc (Fig. 7A). Based on the criteria and algorithm described by Dahiya, the DUSP9 promoter contains a large CpG island near the transcription start site (Fig. 7B). Of note, $11 \mathrm{CpG}$ sites in DUSP9 promoter were involved in this study for bisulfite sequencing analysis (Fig. 7C). The results showed that in normal intestinal mucosa $(n=12)$, DUSP9 promoter showed hypomethylation status (average methylation level 
was $15.4 \%$ ), while in colorectal cancer $(n=12)$, DUSP9 promoter showed hypermethylation status (average methylation level was $87.4 \%, P<0.01$ ) (Fig. 7D). To further determine the relationship between the methylation level and expression level of DUSP9 in CRC, we treated SW480 cells with 5-aza-2'deoxycytidine (5-aza-dC) and examined DUSP9 promoter methylation and protein expression changes. The results showed that with the increase of 5-aza-dC concentration, the methylation level of DUSP9 decreased (Fig. 7E), while the protein expression level increased gradually (Fig. 7F). This suggests that promoter hypermethylation is one of the reasons for low expression of DUSP9 in CRC.

\section{Discussion}

Recently, low expression levels of DUSP9 were reported in a variety of cancers, such as gastric cancer, liver cancer, and renal cancer. In the present study, we systematically investigated the functional role of DUSP9 in CRC and found that DUSP9 was significantly down regulated in tumor tissues compared with peritumor tissues. Moreover, low expression of DUSP9 was correlated with survival time and poor prognosis of patients with CRC. Further statistical analysis revealed that low DUSP9 expression level in CRC was closely associated with tumor size, depth of invasion and advanced TNM stage, indicating that DUSP9 may be involved in the progression of CRC. Functional study revealed that DUSP9 inhibited tumor migration, invasion and metastasis both in vitro and in vivo. Mechanistically, low expression of DUSP9 in CRC was caused by the upregulation of miR-1246. In addition, promoter hypermethylation is another reason for the down-regulation of DUSP9 in CRC.

It has been reported that DUSP9 could affect cell proliferation and participated in the regulation of tumor progression. To our knowledge, this is the first study to report the clinical significance of DUSP9 in CRC. To determine the biological function of DUSP9 in CRC, we performed RNA-seq analysis of gene profiling between CRC cells with DUSP9 knockdown and control cells, and the results revealed that DUSP9 inhibited proliferation and metastasis-related pathways. Next a series of in vitro and in vivo experiments confirmed DUSP9 significantly inhibited the proliferation, invasion and metastasis of CRC cells. However, the molecular mechanism of DUSP9 inhibiting CRC progression has not been fully elucidated. In gastric cancer, low expression level of DUSP9 has been found to be linked with the increased JNK activity and decreased apoptosis, suggesting that DUSP9 may inhibit the proliferation of gastric cancer cells through JNK signaling pathway ${ }^{14}$. In squamous cell carcinoma (SCC), DUSP9 is known to inactivate many members of the mitogen-activated protein (MAP) kinase superfamily (such as SAPK, MAPK, and p38) by dephosphorylating both the phosphotyrosine and phosphoserine/threonine residues ${ }^{15-17}$. The growth

speed of the SCC cells with DUSP9 overexpression got slowly, even to a growth arrest ${ }^{9,}{ }^{18}$. In addition, Wu et al. demonstrated that low expression of the DUSP9 was an independent indicator for poor prognosis of patients with clear cell renal cell carcinomas (ccRCCs) ${ }^{9}$. Moreover, enhanced expression of DUSP9 in malignant tumor cells led to microtubule disruption, cell death and tumor inhibition ${ }^{8,19,20}$. Therefore, DUSP9 may synergistically inhibit the progression of CRC through a variety of mechanisms, which needs to be further verified in future research. 
miRNAs are a class of important small molecule non coding RNA, which regulates target mRNAs at the post-transcriptional level by binding to their 3'-untranslated regions (3-UTRs), resulting in translation inhibition or degradation of mRNAs ${ }^{21}$. MiR-1246 is a frequently upregulated carcinogenic factor and is involved in the invasion, metastasis and chemoresistance in a variety of cancers, including CRC. For instance, Yang et al. demonstrated that miR-1246 promotes metastasis and invasion of lung cancer cells by regulating Wnt/ $\beta$-catenin pathway ${ }^{22}$. Lin et al. reported that miR-1246 was significantly upregulated in oral squamous cell carcinoma (OSCC) tissues and enhanced the stemness hallmarks, which closely associated with cancer relapse and metastasis ${ }^{23}$. In CRC, miR-1246 was also found to be significantly increased in CRC tissues, and functioned as a tumor promoting factor through inducing cell proliferation, migration, invasion and chemoresistance ${ }^{24,25}$. Moreover, some specific exosomal microRNAs (miRNAs), including miR-1246, were identified as a biomarker for metastatic colorectal cancer ${ }^{26-28}$. In addition, Peng et al. demonstrated that miR-1246/SPRED2/MAPK axis played an important role in the progression of $\mathrm{CRC}^{29}$. Consistently, our present study showed that miR-1246 was involved in the downregulation of DUSP9 in CRC. Furthermore, we found that miR-1246 promoted the growth and invasion of CRC cells by inhibiting DUSP9. Therefore, miR-1246/DUSP9 axis might be a promising strategy for CRC treatment.

Methylation of $\mathrm{CpG}$ island in the promoter region of tumor suppressor genes can promote the initiation of many cancers, including CRC. Moreover, the de novo methylation of genes seems to be common event in most malignancies ${ }^{30-33}$. In many types of cancer, hypermethylation of $\mathrm{CpG}$ island in promoter of tumor suppressor genes is an important event. It can affect genes related to cell cycle, apoptosis, DNA repair, angiogenesis, etc ${ }^{14,34,35}$. In the present study, we used MethPrimer software to predict the methylation status in DUSP9 gene promoter and found that DUSP9 was hypermethylated in a variety of cancers, such as colon adenocarcinoma (COAD), bladder urothelial carcinoma (BLCA), breast invasive carcinoma (BRCA), lung adenocarcinoma (LUAD) and pancreatic adenocarcinoma et al. Furthermore, bisulfite sequencing analysis revealed the hypermethylation status of $\mathrm{CpG}$ island in promoter of DUSP9, which further led to a significant decrease of DUSP9 expression levels in human CRC. As far as we know, this is the first study using bisulfite sequencing analysis for methylation status analysis of DUSP9 in clinical human CRC samples.

\section{Conclusions}

The results of this study indicate that down-regulated DUSP9 promotes proliferation, invasion and metastasis of tumor cells and associated with poor prognosis of patients with CRC. Mechanistically, low expression of DUSP9 in CRC was caused by the upregulation of miR-1246 and hypermethylation status of $\mathrm{CpG}$ island in promoter of DUSP9 (Fig. 8). This finding suggests that therapeutic intervention to increase the expression or activity of DUSP9 may be a potential therapeutic target for CRC treatment in the future.

\section{Abbreviations}




\begin{tabular}{ll} 
DUSP9 & Dual-specificity phosphatase 9 \\
\hline CRC & Colorectal cancer \\
\hline BSE & Bisulfite sequencing \\
\hline MKP-4 & Mitogen-activated protein kinase phosphatase 4 \\
\hline NAFLD & Nonalcoholic fatty liver disease \\
\hline NSCLC & Non-small cell lung cancer \\
\hline qRT-PCR & Quantitative real-time reverse transcription PCR \\
\hline OS & Overall survival \\
\hline DFS & Disease-free survival \\
\hline DEGs & Differently expressed genes \\
\hline EMT & Epithelial-mesenchymal transition \\
\hline COAD & Colon adenocarcinoma \\
\hline BLCA & Bladder urothelial carcinoma \\
\hline BRCA & Breast invasive carcinoma \\
\hline CESC & Cervical squamous cells carcinoma \\
\hline LUAD & Lung adenocarcinoma \\
\hline SCC & Squamous cell carcinoma \\
\hline cCRCCs & Clear cell renal cell carcinomas \\
\hline 3'-UTR & 3'-untranslated regions \\
\hline miRNAs & MicroRNAs \\
\hline OSCC & Oral squamous carcinoma \\
\hline OSC
\end{tabular}

\section{Declarations}

\section{Authors' contributions}

Z. Y. Q. and Q. W. designed the study. Z. Y. Q., N. L. wrote the paper. T. S. and H. Y. X. performed the experiment. Z. Y. Q., Q. W. and T. Y. X. analyzed the data. X. X. W. helped revised the manuscript. All authors read and approved the final manuscript.

\section{Funding}


This study was funded by grants from National Center for clinical medicine of geriatric diseases of China (No. NCRCG-PLAGH-2018005).

\section{Availability of supporting data}

The data analyzed in this study are available from the corresponding author on reasonable request.

\section{Ethics approval and consent to participate}

All patients involved in this study gave their informed consent. Institution review board approval of our hospital was obtained for this study. The animal experiments complied with the policy of PLAGH on the care and used of laboratory animals.

\section{Consent for publication}

Not applicable.

\section{Acknowledgments}

We thank Dr. Yixun Lu for helping us revise the manuscript.

\section{Competing interests}

The authors declare no competing financial interests.

\section{Contributor information}

Zhaoyan Qiu, Email: 301qzy@sina.com.

Ning Liang, Email: 303374695@qq.com

Tao Sun, Email: 673424181@qq.com

Hongyuan Xue, Email: xuehongyuan2011@163.com

Tianyu Xie, Email: xty930214@163.com

Xinxin Wang, Email: 301wxx@sina.com

Qian Wang, Email: 1211072068@qq.com.

\section{References}

1. Jemal A, Bray F, Center MM, et al. Global cancer statistics. CA Cancer J Clin 2011;61:69-90.

2. Miller KD, Siegel RL, Lin CC, et al. Cancer treatment and survivorship statistics, 2016. CA Cancer J Clin 2016;66:271-89. 
3. Dekker E, Tanis PJ, Vleugels JLA, et al. Colorectal cancer. Lancet 2019;394:1467-1480.

4. Brenner H, Kloor M, Pox CP. Colorectal cancer. Lancet 2014;383:1490-1502.

5. Farooq A, Zhou MM. Structure and regulation of MAPK phosphatases. Cell Signal 2004;16:769-79.

6. Christie GR, Williams DJ, Macisaac F, et al. The dual-specificity protein phosphatase DUSP9/MKP-4 is essential for placental function but is not required for normal embryonic development. Mol Cell Biol 2005;25:8323-33.

7. Ye P, Xiang M, Liao H, et al. Dual-Specificity Phosphatase 9 Protects Against Nonalcoholic Fatty Liver Disease in Mice Through ASK1 Suppression. Hepatology 2019;69:76-93.

8. Liu Y, Lagowski J, Sundholm A, et al. Microtubule disruption and tumor suppression by mitogenactivated protein kinase phosphatase 4 . Cancer Res 2007;67:10711-9.

9. Wu S, Wang Y, Sun L, et al. Decreased expression of dual-specificity phosphatase 9 is associated with poor prognosis in clear cell renal cell carcinoma. BMC Cancer 2011;11:413.

10. Jenner S, Wiedorn KH, Techel D. Development of a DUSP9 methylation screening assay. Pathol Oncol Res 2015;21:123-30.

11. Liu J, Ni W, Xiao M, et al. Decreased expression and prognostic role of mitogen-activated protein kinase phosphatase 4 in hepatocellular carcinoma. J Gastrointest Surg 2013;17:756-65.

12. Keyse SM. Dual-specificity MAP kinase phosphatases (MKPs) and cancer. Cancer Metastasis Rev 2008;27:253-61.

13. Wang Q, Wang G, Wang $Y$, et al. Association of AlkB homolog 3 expression with tumor recurrence and unfavorable prognosis in hepatocellular carcinoma. J Gastroenterol Hepatol 2018.

14. Wu F, Lv T, Chen G, et al. Epigenetic silencing of DUSP9 induces the proliferation of human gastric cancer by activating JNK signaling. Oncol Rep 2015;34:121-8.

15. Lewis TS, Shapiro PS, Ahn NG. Signal transduction through MAP kinase cascades. Adv Cancer Res 1998;74:49-139.

16. Brondello JM, Brunet A, Pouyssegur $\mathrm{J}$, et al. The dual specificity mitogen-activated protein kinase phosphatase- 1 and -2 are induced by the p42/p44MAPK cascade. J Biol Chem 1997;272:1368-76.

17. Sun H, Tonks NK, Bar-Sagi D. Inhibition of Ras-induced DNA synthesis by expression of the phosphatase MKP-1. Science 1994;266:285-8.

18. Keyse SM. Protein phosphatases and the regulation of mitogen-activated protein kinase signalling. Curr Opin Cell Biol 2000;12:186-92.

19. Solit DB, Garraway LA, Pratilas CA, et al. BRAF mutation predicts sensitivity to MEK inhibition. Nature 2006;439:358-62.

20. Liao Q, Guo J, Kleeff J, et al. Down-regulation of the dual-specificity phosphatase MKP-1 suppresses tumorigenicity of pancreatic cancer cells. Gastroenterology 2003;124:1830-45.

21. Lei C, Du F, Sun L, et al. miR-143 and miR-145 inhibit gastric cancer cell migration and metastasis by suppressing MYO6. Cell Death Dis 2017;8:e3101. 
22. Yang F, Xiong H, Duan L, et al. MiR-1246 Promotes Metastasis and Invasion of A549 cells by Targeting GSK-3betaMediated Wnt/beta-Catenin Pathway. Cancer Res Treat 2019;51:1420-1429.

23. Lin SS, Peng CY, Liao YW, et al. miR-1246 Targets CCNG2 to Enhance Cancer Stemness and Chemoresistance in Oral Carcinomas. Cancers (Basel) 2018;10.

24. Wang S, Zeng Y, Zhou JM, et al. MicroRNA-1246 promotes growth and metastasis of colorectal cancer cells involving CCNG2 reduction. Mol Med Rep 2016;13:273-80.

25. Jin G, Liu Y, Zhang J, et al. A panel of serum exosomal microRNAs as predictive markers for chemoresistance in advanced colorectal cancer. Cancer Chemother Pharmacol 2019;84:315-325.

26. Tang $Y$, Zhao Y, Song $X$, et al. Tumor-derived exosomal miRNA-320d as a biomarker for metastatic colorectal cancer. J Clin Lab Anal 2019;33:e23004.

27. Eylem CC, Yilmaz M, Derkus B, et al. Untargeted multi-omic analysis of colorectal cancer-specific exosomes reveals joint pathways of colorectal cancer in both clinical samples and cell culture. Cancer Lett 2020;469:186-194.

28. Lai X, Friedman A. Exosomal microRNA concentrations in colorectal cancer: A mathematical model. J Theor Biol 2017;415:70-83.

29. Peng W, Li J, Chen R, et al. Upregulated METTL3 promotes metastasis of colorectal Cancer via miR1246/SPRED2/MAPK signaling pathway. J Exp Clin Cancer Res 2019;38:393.

30. Baylin SB, Herman JG, Graff JR, et al. Alterations in DNA methylation: a fundamental aspect of neoplasia. Adv Cancer Res 1998;72:141-96.

31. Palau A, Perucho M, Esteller M, et al. First Barcelona Conference on Epigenetics and Cancer. Epigenetics 2014;9:468-75.

32. Liu G, Liu YJ, Lian WJ, et al. Reduced BMP6 expression by DNA methylation contributes to EMT and drug resistance in breast cancer cells. Oncol Rep 2014;32:581-8.

33. Minning C, Mokhtar NM, Abdullah N, et al. Exploring breast carcinogenesis through integrative genomics and epigenomics analyses. Int J Oncol 2014;45:1959-68.

34. Esteller M. Epigenetics in cancer. N Engl J Med 2008;358:1148-59.

35. Guerrero-Preston R, Hadar T, Ostrow KL, et al. Differential promoter methylation of kinesin family member $1 \mathrm{a}$ in plasma is associated with breast cancer and DNA repair capacity. Oncol Rep 2014;32:505-12.

\section{Table}

Table 1. Relationship between tumor DUSP9 expression and clinic features. 


\begin{tabular}{|c|c|c|c|c|}
\hline \multirow[t]{2}{*}{ Variables } & \multirow[t]{2}{*}{ No. of cases } & \multicolumn{2}{|c|}{ DUSP9 expression } & \multirow[t]{2}{*}{$P$ value } \\
\hline & & Low & High & \\
\hline All & 245 & 154 & 91 & \\
\hline Age & & & & 0.791 \\
\hline$<60$ & 117 & 75 & 42 & \\
\hline$>=60$ & 128 & 79 & 49 & \\
\hline Gender & & & & 0.792 \\
\hline Female & 113 & 70 & 43 & \\
\hline Male & 132 & 84 & 48 & \\
\hline Tumor site & & & & 0.181 \\
\hline colon & 142 & 84 & 58 & \\
\hline Rectum & 103 & 70 & 33 & \\
\hline Tumor size & & & & 0.001 \\
\hline$<5.0 \mathrm{~cm}$ & 115 & 60 & 55 & \\
\hline$>=5 \mathrm{~cm}$ & 130 & 94 & 36 & \\
\hline Differentiation grade & & & & 0.219 \\
\hline well & 154 & 92 & 62 & \\
\hline Poor & 91 & 62 & 29 & \\
\hline Depth of invasion & & & & 0.003 \\
\hline $\mathrm{T} 1+\mathrm{T} 2$ & 112 & 59 & 53 & \\
\hline $\mathrm{T} 3+\mathrm{T} 4$ & 133 & 95 & 38 & \\
\hline Lymph node metastasis & & & & 0.227 \\
\hline Absent & 107 & 97 & 50 & \\
\hline Present & 138 & 57 & 41 & \\
\hline Distant metastasis & & & & 0.409 \\
\hline Absent & 157 & 102 & 55 & \\
\hline Present & 88 & 52 & 36 & \\
\hline TNM stage & & & & 0.005 \\
\hline I+ II & 108 & 57 & 51 & \\
\hline III+ IV & 137 & 97 & 40 & \\
\hline
\end{tabular}

Figures 


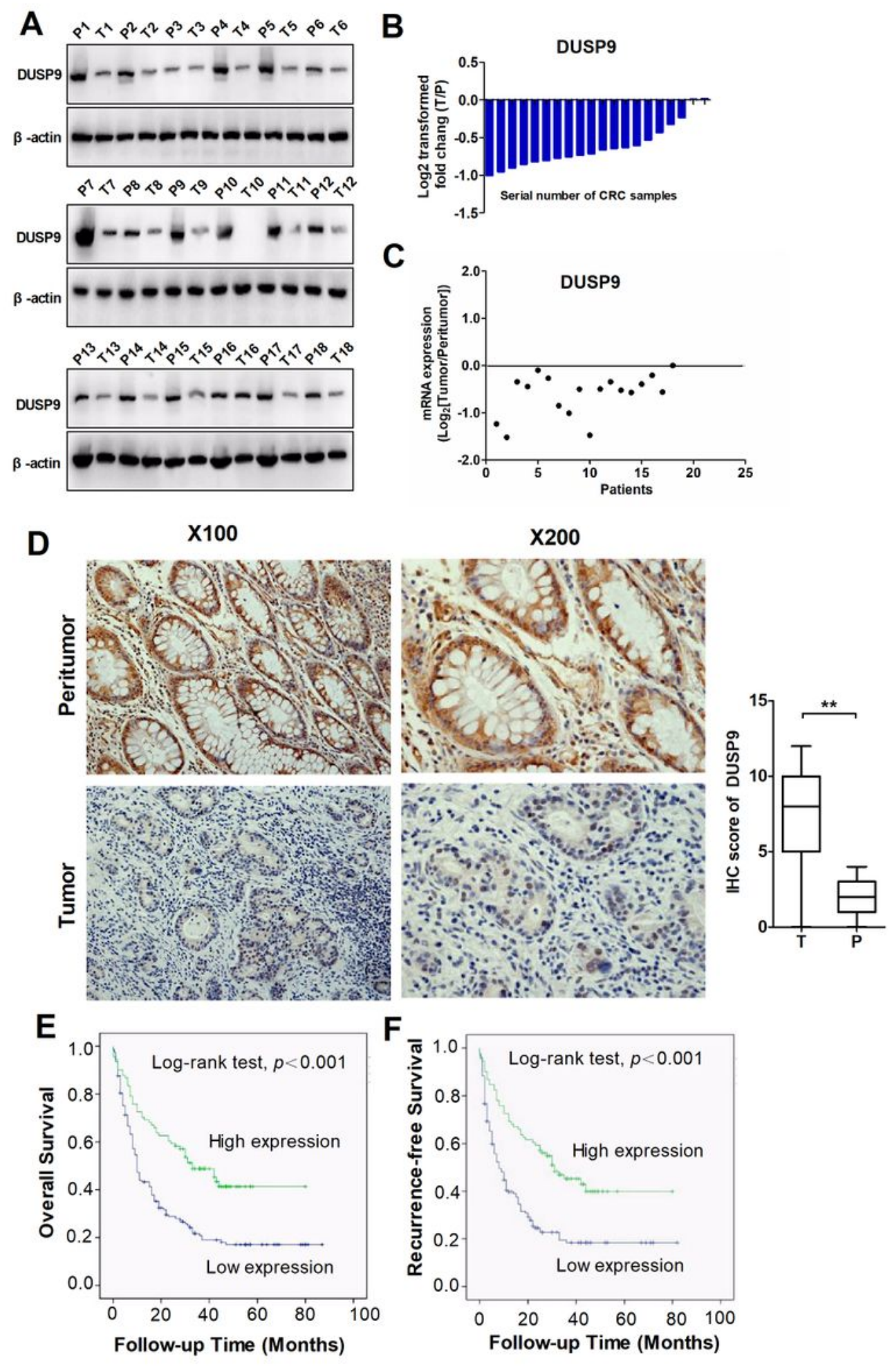

\section{Figure 2}

DUSP9 is down regulated in CRC tissues and associated with tumor progression and poor prognosis. (A) The protein expression levels of DUSP9 in 18 paired CRC and peritumor tissues were performed by western blot. T, tumor; P, peritumor. (B) Protein quantitative results of western blot. (C) The mRNA expression levels of DUSP9 in 18 paired CRC and peritumor tissues were performed by qRT-PCR analysis. (D) Immunohistochemical (IHC) staining analysis of DUSP9 expression in paired CRC and peritumor 
tissues $(n=245) .{ }^{*} P<0.01$. (E,F) Kaplan-Meier survival curves for overall survival (OS) and recurrencefree survival (RFS) stratified by DUSP9 expression in 245 tumor issues from CRC patients.
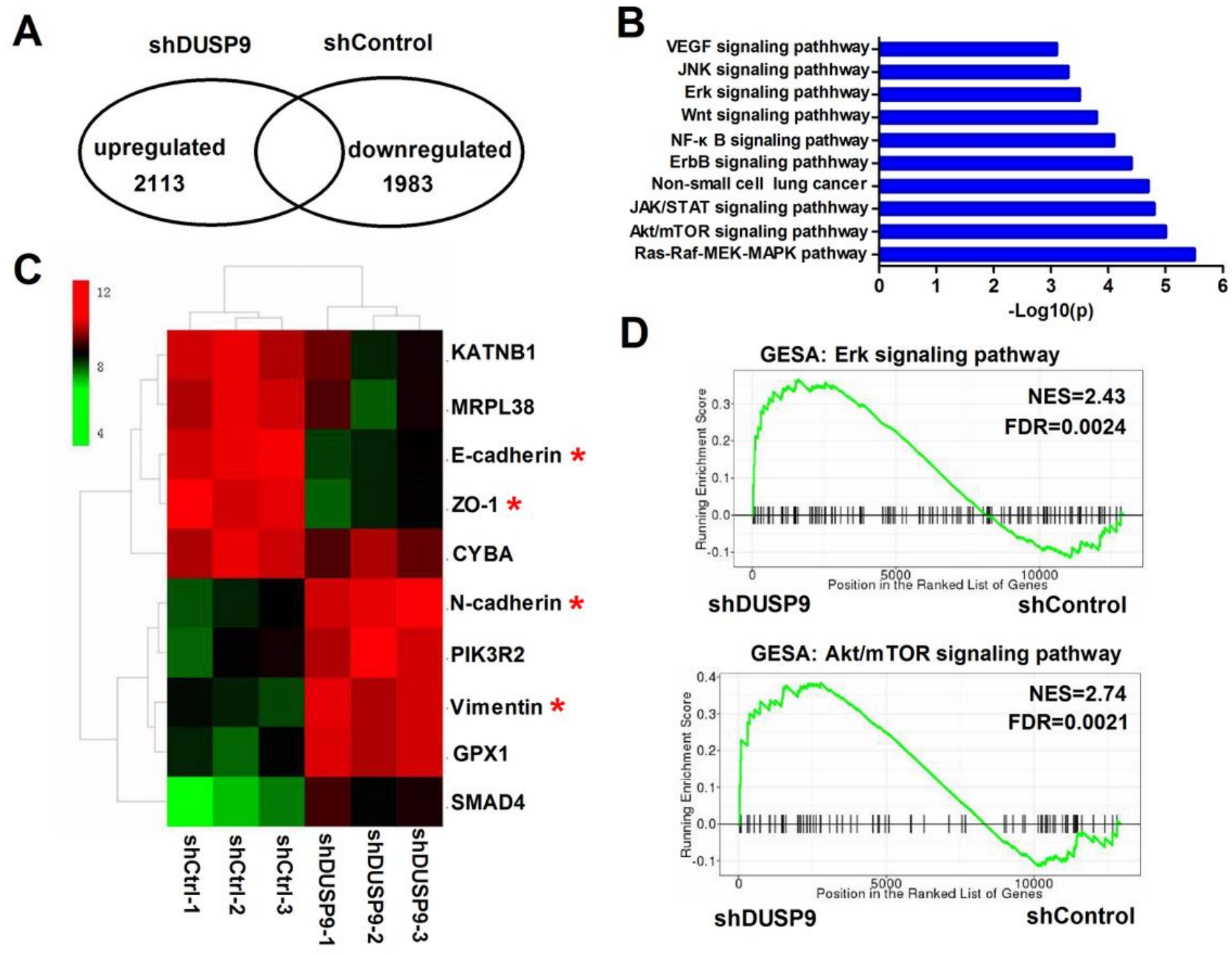

D

GESA: Erk signaling pathway

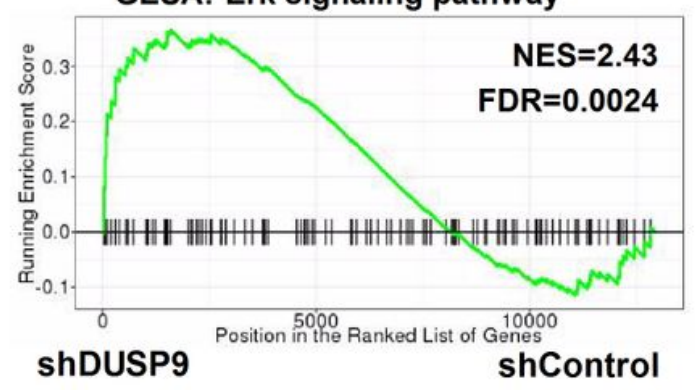

GESA: Akt/mTOR signaling pathway

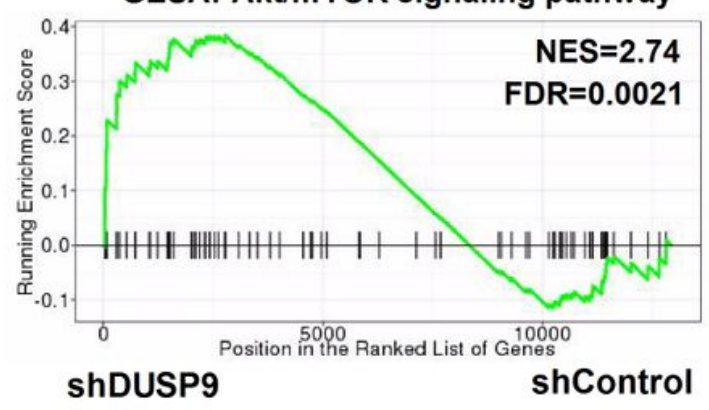

Figure 4

RNA-seq revealed that DUSP9 inhibited proliferation and metastasis-related pathways in CRC. (A) Dysregulated genes between SW480 cells with DUSP9 stable knockdown and SW480-shControl cells. Of the 4096 dysregulated genes between two groups, 2113 genes were up-regulated (fold change $\geq 2$ ) and 1983 genes were down-regulated (fold change $\geq 2$ ). (B) Top 10 KEGG pathway enriched by the differentially expressed genes. (C) Cluster analysis of DEGs annotated in pathways associated with epithelial mesenchymal transformation (EMT). (D) GSEA (Gene Set Enrichment Analysis) shows that DUSP9 knockdown will lead to the activation of Erk and Akt/mTOR signaling pathway. 


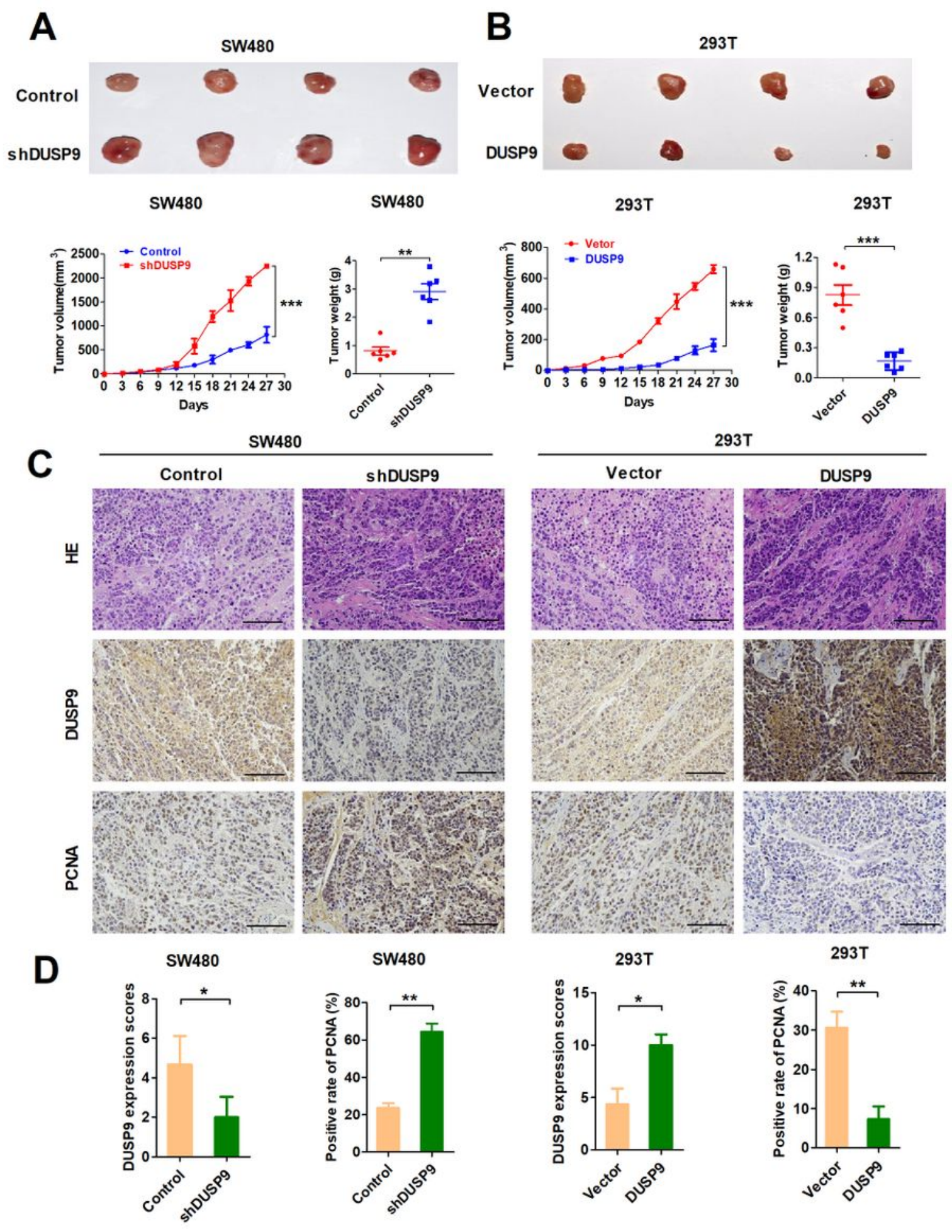

\section{Figure 5}

DUSP9 suppresses tumor growth in vivo. (A) DUSP9 knockdown promoted growth of SW480 xenograft tumors, which were generated by injecting SW480-shDUSP9 cells or SW480-Control cells. The growth of xenograft tumors was measured by volume, and the tumor weight was measured. (B) DUSP9 overexpression inhibited growth of 293T xenograft tumors, which were generated by injecting 293T cells overexpressing DUSP9 or carrying a control vector. The growth of xenograft tumors was measured by 
volume, and the tumor weight was measured. (C, D) HE, IHC, PCNA staining of the xenograft tumors are shown. ${ }^{*} \mathrm{P}<0.05,{ }^{*} \mathrm{P}<0.01$.
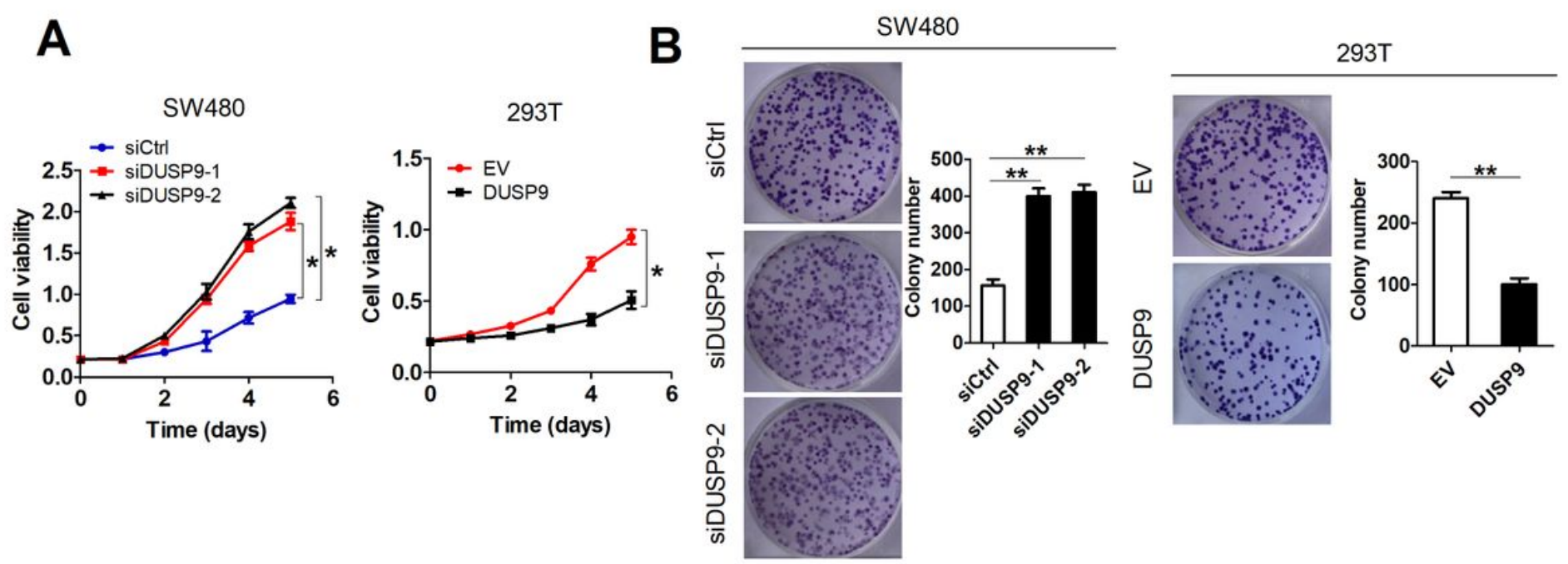

C

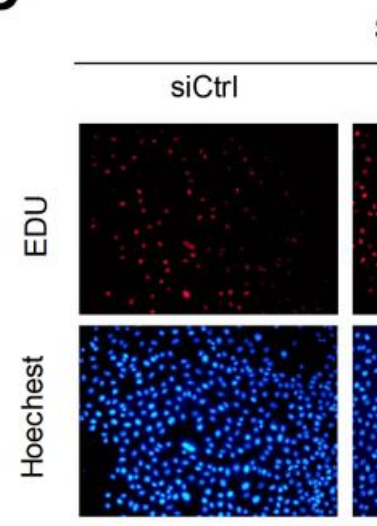

SW480

$$
\text { siDUSP9 }
$$

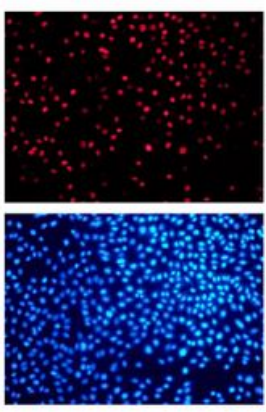

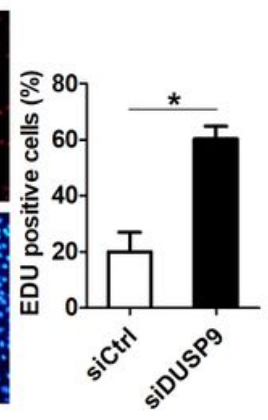
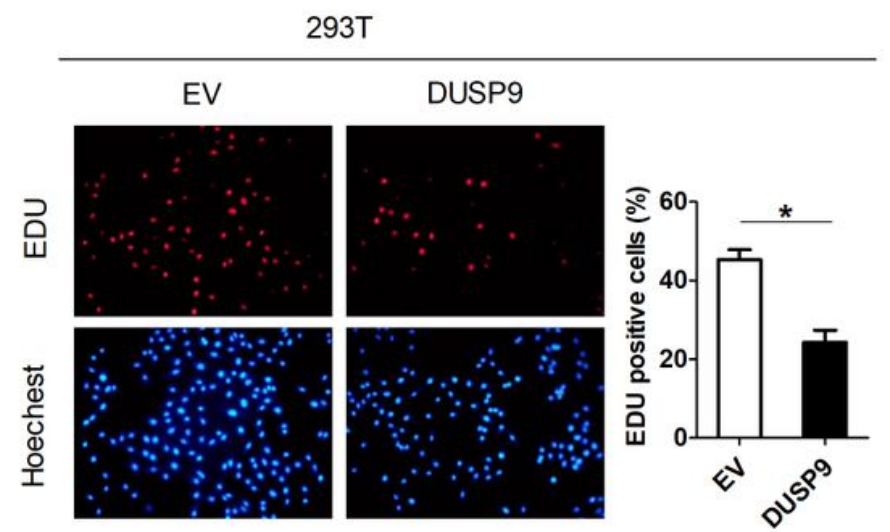

Figure 7

Enforced expression of DUSP9 inhibits proliferation of CRC cells. (A) The MTS cell viability assay was performed in SW480 and 293T cells with the indicated treatments (siDUSP9-1 and siDUSP9-2: siRNAs against DUSP9; siCtrl: control siRNA; DUSP9: expression vector encoding DUSP9; EV: empty vector). (B) Colony formation assay in SW480 and 293T cells with treatments as indicated. (C) EdU incorporation assay in SW480 and 293T cells with treatments as indicated. 
A

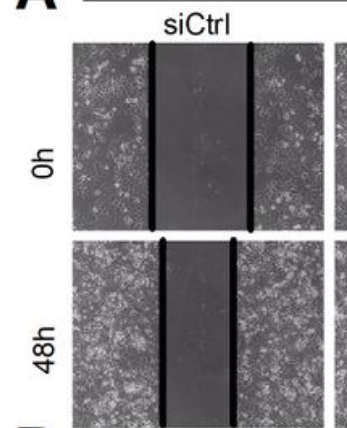

B

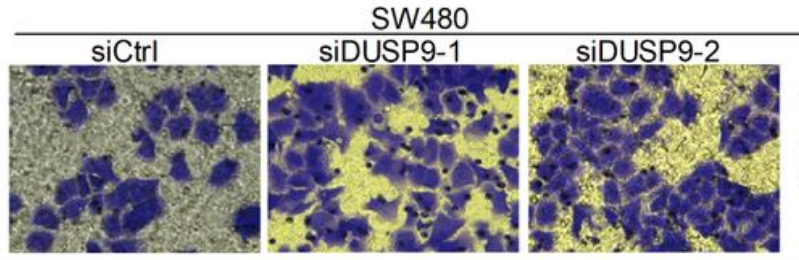

C

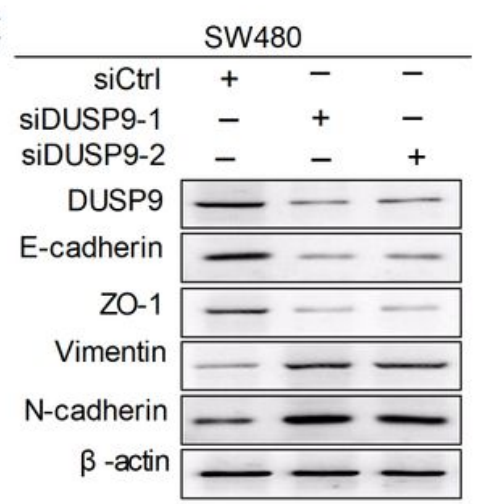

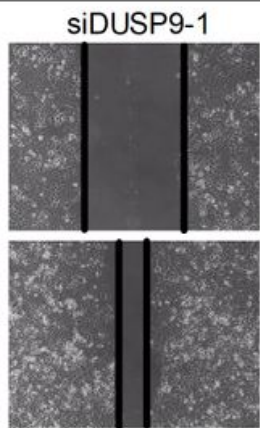
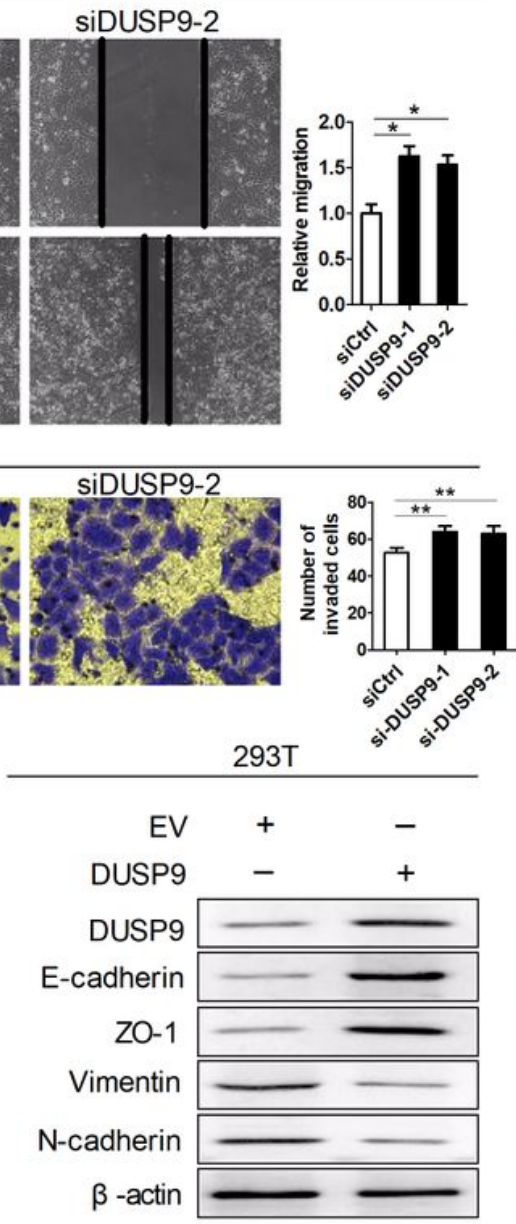

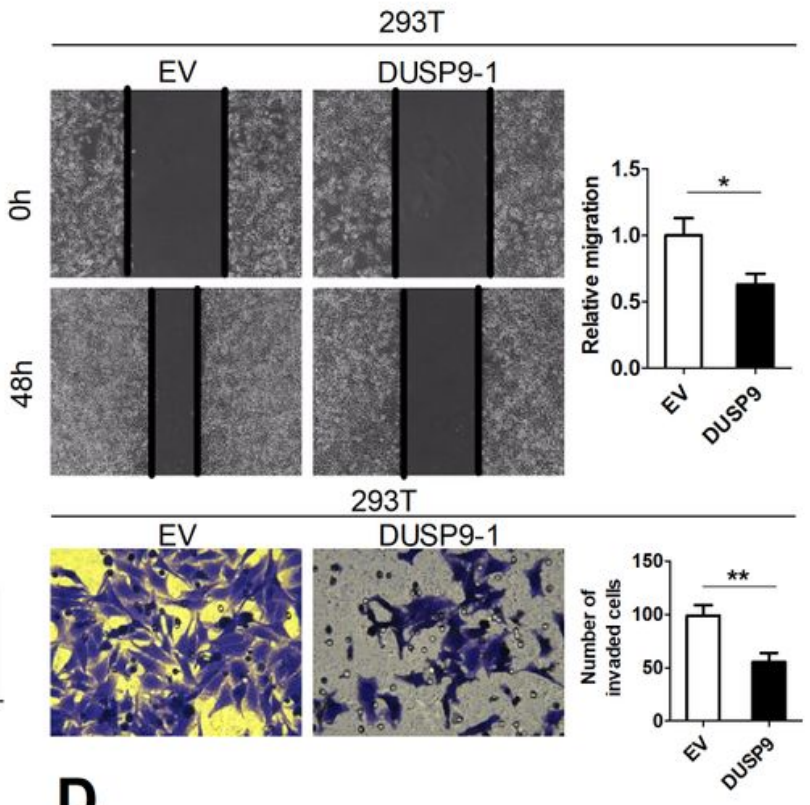

D
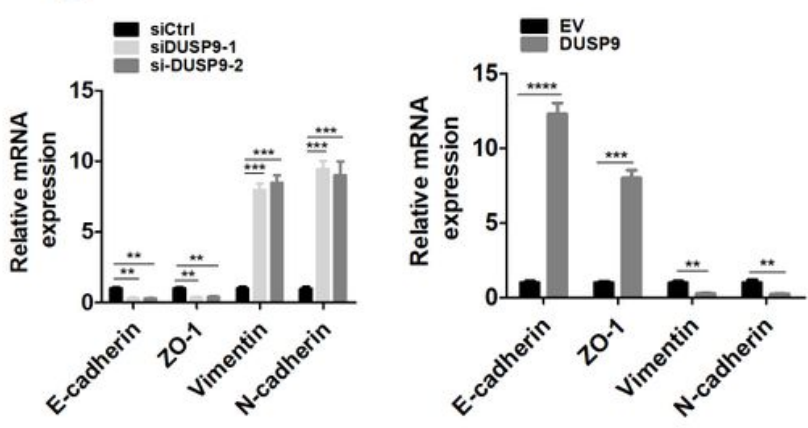

Figure 10

DUSP9 inhibits tumor migration, invasion and metastasis in vitro. (A) Scratch wound healing assays for cell migration abilities in SW480 and 293T cells with the indicated treatments. (B) Transwell invasion assays for cell invasion abilities in SW480 and 293T cells with the indicated treatments. (C, D) Western blot and qRT-PCR analyses for expressions of Epithelial-mesenchymal transition (EMT)-related markers in both SW480 and 293T cells with treatment as indicated. ${ }^{\star *} P<0.01, * \star \star P<0.001,{ }^{\star} * \star * P<0.0001$. 
A

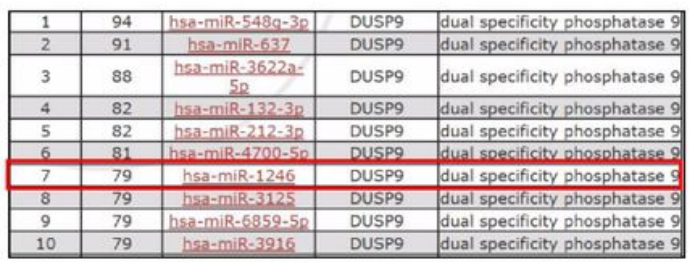

D

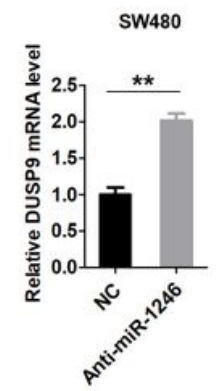

$\mathbf{E}$

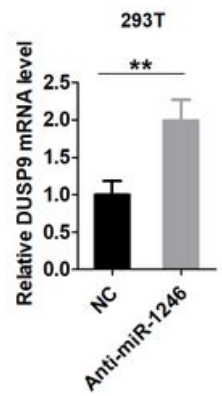

B

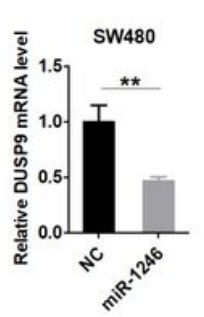

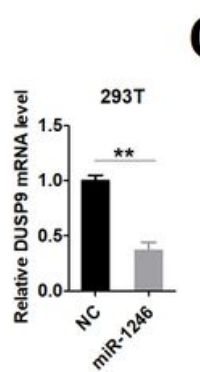

C
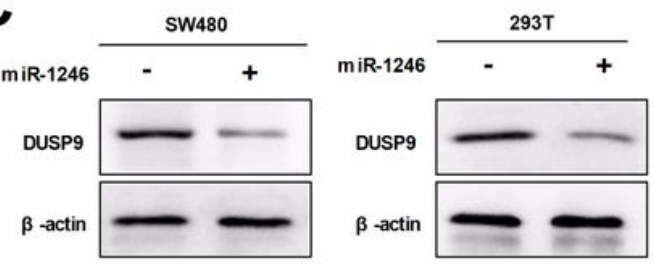

$\mathbf{F}$
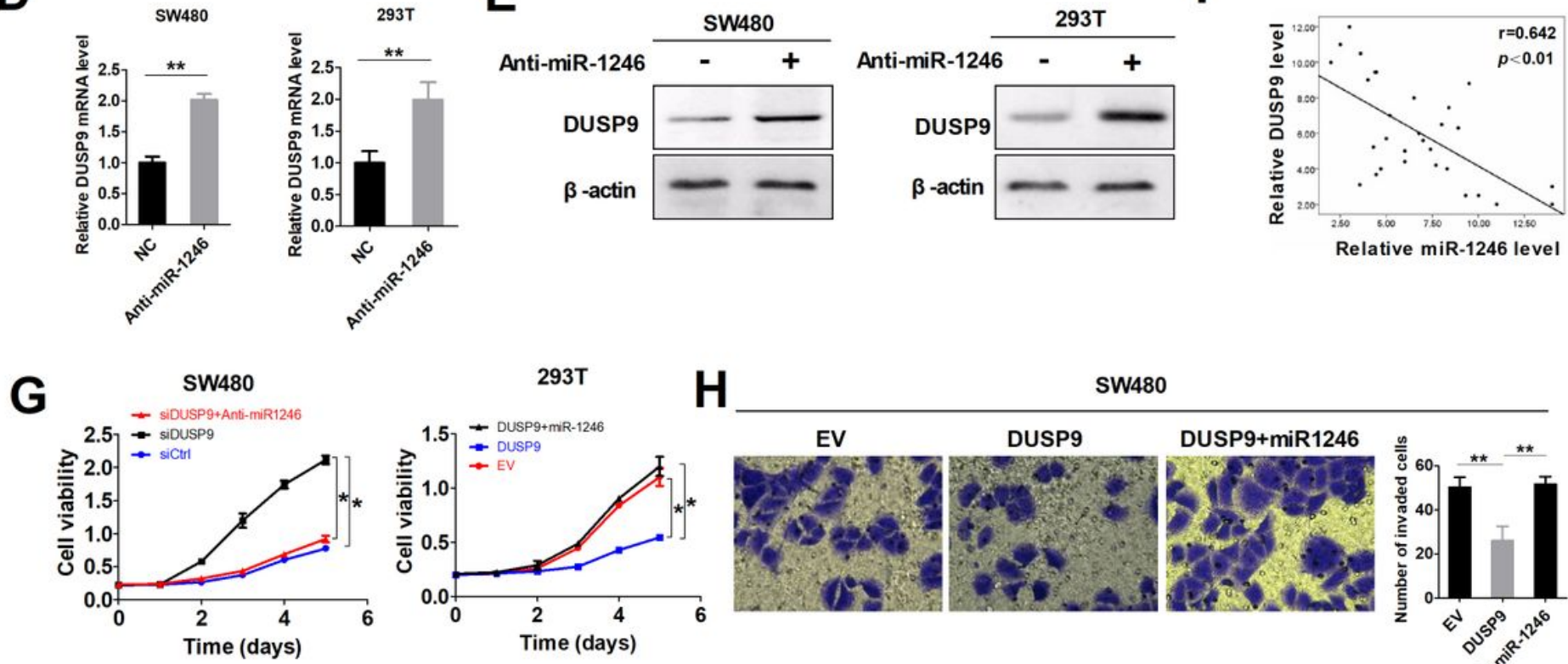

$\mathbf{H}$

SW480
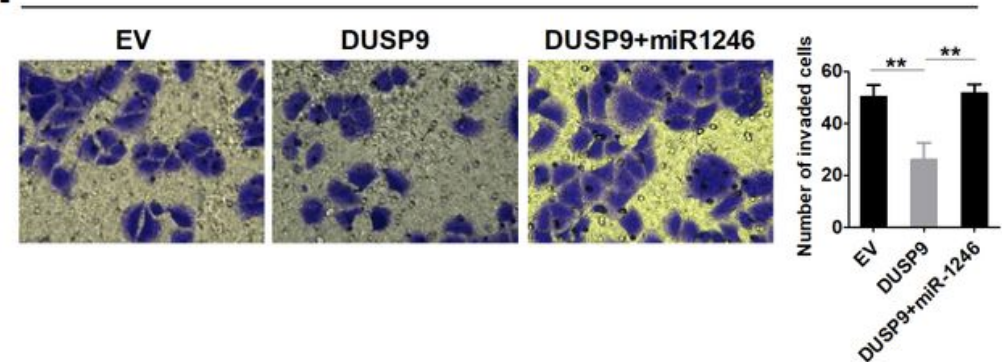

Figure 12

Low expression of DUSP9 is mainly mediated by upregulation of miR-1246. (A) The top ten predicted miRNAs targeting DUSP9 were identified using MicroRNA Data Integration Portal (mirDIP)-based target prediction programs. (B) qRT-PCR and (C) western blot analysis for DUSP9 expression in SW480 and 293T cells transfected with the miR-1246 mimics. (D) qRT-PCR and (E) western blot analysis for DUSP9 expression in SW480 and 293T cells transfected with the miR-1246 inhibitor (Anti-miR1246). (F) The correlation between the mRNA levels of DUSP9 and miR-1246 was determined. (G) MTS cell viability assays in SW480 and 293T cells with treatment as indicated. $(\mathrm{H})$ Transwell matrigel invasion assays in SW480 cells with treatment as indicated. ${ }^{*} \mathrm{P}<0.05,{ }^{\star *} \mathrm{P}<0.01$. 

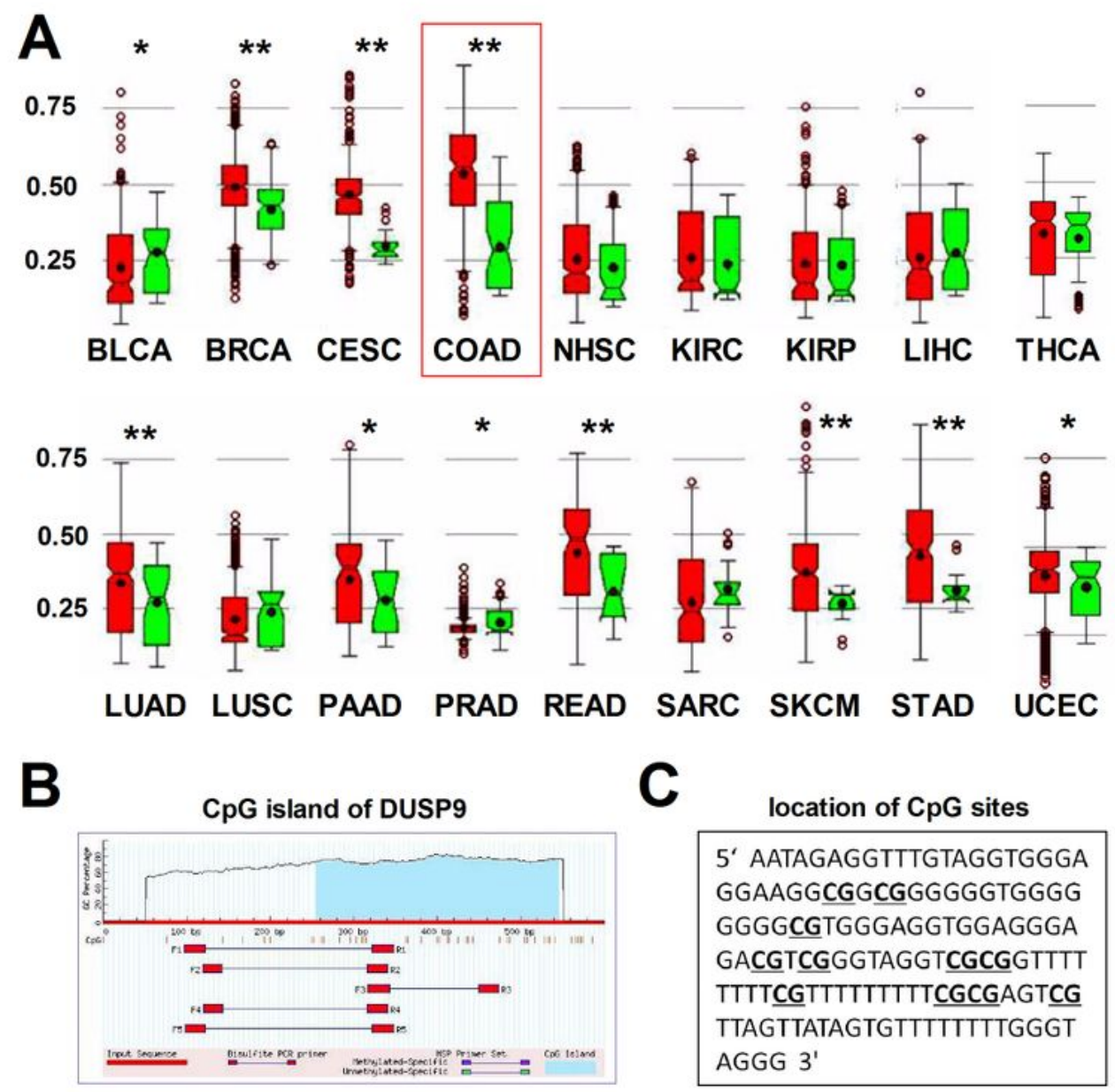

5' AATAGAGGTTTGTAGGTGGGA GGAAGGCGGCGGGGGGTGGGG GGGGCGTGGGAGGTGGAGGGA GACGTCGGGTAGGTCGCGGTTTT TTTTCGTTTTTTTTTCGCGAGTCG TTAGTTATAGTGTTTTTTTTGGGT AGGG $3^{\prime}$

D DUSP9 promoter methylation status

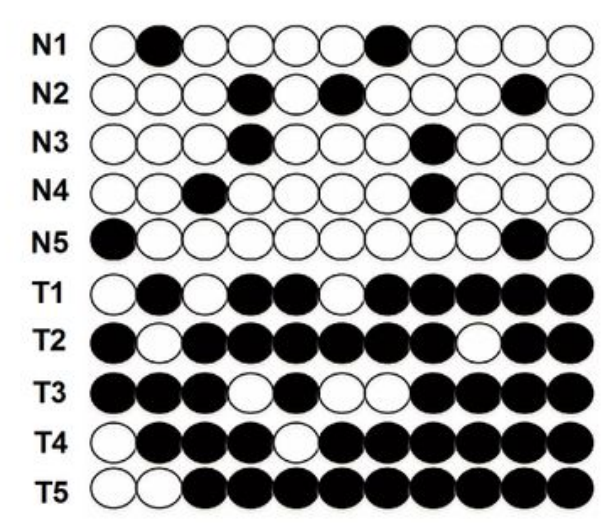

E

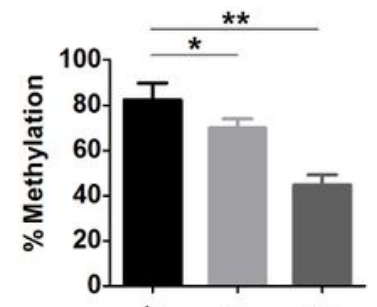

F
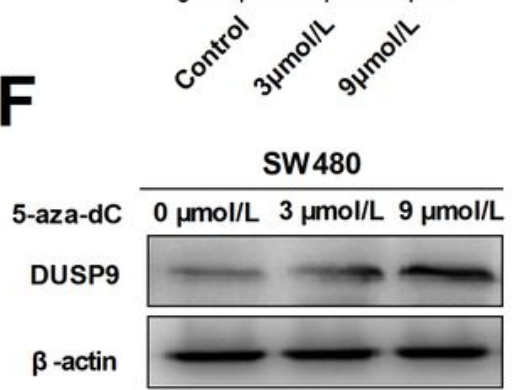

Figure 14

DUSP9 expression is silenced via promoter hypermethylation in CRC. (A) MethPrimer software was used to predict the methylation status of DUSP9 gene promoter in a variety of cancers, including bladder urothelial carcinoma (BLCA), breast invasive carcinoma (BRCA), cervical squamous cell carcinoma and endocervical adenocarcinoma (CESC), colon adenocarcinoma (COAD), head and neck squamous cell carcinoma (NHSC), kidney renal clear cell carcinoma (KIRC), kidney renal papillary cell carcinoma (KIRP), 
liver hepatocellular carcinoma (LIHC), thyroid carcinoma (THCA), lung adenocarcinoma (LUAD), Lung squamous cell carcinoma (LUSC), pancreatic adenocarcinoma (PAAD), Prostate adenocarcinoma (PRAD), rectum adenocarcinoma (READ), sarcoma (SARC), skin cutaneous Melanoma (SKCM), stomach adenocarcinoma (STAD), uterine corpus endometrial carcinoma (UCEC). ${ }^{*} P<0.05, * * P<0.01$. (B) Schematic representation of the human DUSP9 promoter. Each red tick mark represents is one CpG site. (C) Location of $11 \mathrm{CpG}$ sites in the DUSP9 promoter. (D) Bisulfite sequencing evaluation of $\mathrm{CpG}$ island methylation of the $11 \mathrm{CpG}$ sites of DUSP9 promoter in CRC ( $T=12)$ and normal intestinal mucosa $(\mathrm{N}=12)$. White spots, unmethylated $\mathrm{CpG}$; black spots, methylated $\mathrm{CpG}$. (E) Bisulfite sequencing was carried out on $11 \mathrm{CpG}$ sites in SW480 cells following treatment with 5 -aza-dC for $72 \mathrm{~h}$ to determine the methylation level. ${ }^{*} \mathrm{P}<0.05,{ }^{*} \mathrm{P}<0.01$. (F) Treatment with 5 -aza-dC can lead to DNA demethylation and restore DUSP9 expression in SW480 cells.

\section{Normal Colorectal Cells}
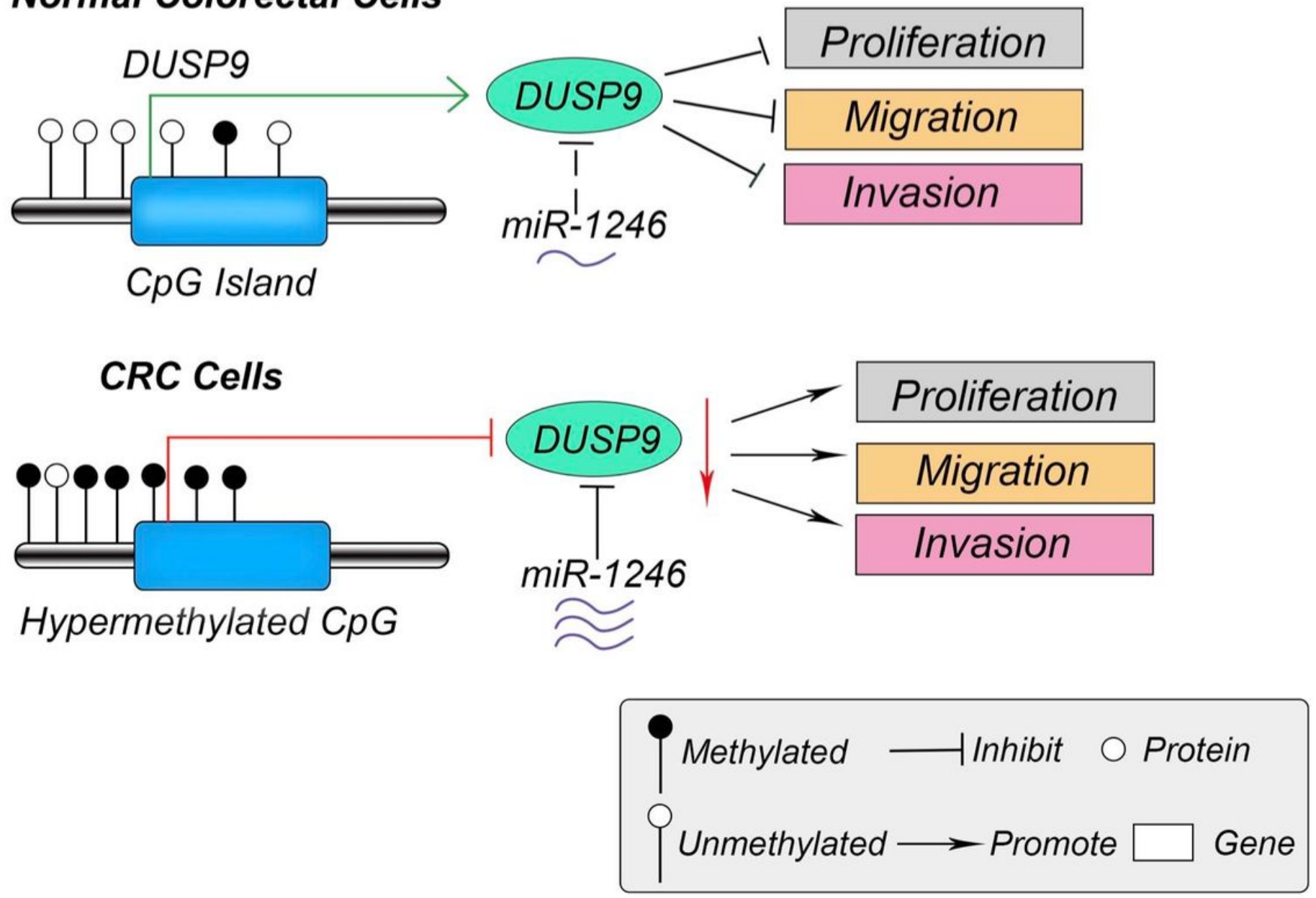

Figure 16

The schematic diagram of DUSP9 in CRC. In normal colorectal cells, the promoter of DUSP9 is hypomethylated, and the expression level of DUSP9 is relatively high, which inhibits cell proliferation, migration and infiltration. However, the hypermethylation status of $\mathrm{CpG}$ island in promoter of DUSP9 could lead to a significant decrease of DUSP9 expression levels in human CRC. Moreover, the increased 
miR-1246 in CRC further reduced the expression level of DUSP9, which promoted tumor cells proliferate, migrate and invade. CRC: colorectal cancer.

\section{Supplementary Files}

This is a list of supplementary files associated with this preprint. Click to download.

- Supplementaltable3.docx

- Supplementaltable2.docx

- Supplementaltable2.docx

- Supplementaltable3.docx

- Supplementaltable1.docx

- Supplementaltable1.docx 